\title{
Alphablocker erhöhen das Sturzrisiko leicht
}

Bei Patienten mit hohem Sturzrisiko rät man in der Regel von Alphablockern ab, da sie eine Hypotonie auslösen können. Diesen Grundsatz unterstützt nun eine retrospektive Analyse der Daten von 147.000 Männern mit benigner Prostatahyperplasie, die zwischen 2003 und 2013 eine The- rapie begonnen hatten. Die Einnahme von Alphablockern war dabei mit einem etwa $12 \%$ erhöhten Sturzrisiko assoziiert. $\mathrm{Zu}$ hypotonen Notfällen kam es doppelt so oft wie bei Patienten, die ohne Alphablocker behandelt wurden.

- BMJ 2015;351:h5398

\section{Ein sanfter Tod}

\section{Hausärztlich unterstütztes Sterbefasten}

Schwerkranke Menschen versuchen nicht selten, ihren Tod, den sie als Erlösung empfinden, schneller herbeizuführen, indem sie bewusst auf Essen und Trinken verzichten. Ursachen sind zumeist Fatigue, Verschlechterung des Befindens oder Schmerzen ohne Hoffnung auf Besserung. Der Tod tritt in diesen Fällen einer niederländischen Studie zufolge im Median innerhalb von sieben
Tagen ein. Die Autoren halten dies für einen relativ sanften Weg, das Sterben zu beschleunigen - eine gute palliativmedizinische Versorgung vorausgesetzt. Hier könne der Hausarzt helfen, indem er darauf achtet, dass die letzten Tage nicht durch Mundtrockenheit, Schmerzen, Delir oder Agitiertheit erschwert würden.

- Ann Fam Med 2015;13:421-8

\section{Diät-Studie}

\section{Weniger Fett ist nicht gleich weniger Speck}

Wo sich viel Fett auf die Rippen gelegt hat, sollte wenig Fett konsumiert werden - das klingt zunächst plausibel. An entsprechenden Low-Fat-Diäten herrscht ebenso wenig ein Mangel wie an Untersuchungen, in denen ihren Effekten nachgegangen wurde.
An der Harvard-Universität haben Forscher 53 Ernährungsstudien in randomisiert-kontrolliertem Design daraufhin analysiert, ob Patienten unter Low-FatDiäten mehr Gewicht verlieren. Alle untersuchten Interventionen dauerten mindestens ein Jahr.

Verglichen mit fetthaltigeren Diäten führten die Low-Fat-Regime nach einem Jahr nur zu einem zusätzlichen Gewichtsverlust von $360 \mathrm{Gramm}$. Statistisch war das irrelevant. Probanden, die auf wenig Kohlenhydrate setzen (Low-Carb-Diät), schnitten dagegen besser ab. Sie verloren im Mittel 1,15 kg mehr als die Low-FatJünger.

- Lancet Diabetes Endocrinol 2015, online 30. Oktober; doi: 10.1016/\$2213-8587(15)00367-8
Dr. med. Dirk Einecke Chefredakteur dirk.einecke@springer.com

\section{Bursitis olecrani}

Konservativ ist besser

Die Gewohnheit, beim Lesen (auch vor dem Computerbildschirm) den Ellenbogen aufzustützen, genügt schon: Der Schleimbeutel am Olecranon entzündet sich.

Wie man die Beschwerden am besten lindert, haben koreanische Orthopäden untersucht. Sie behandelten jeweils $30 \mathrm{~Pa}$ tienten entweder eine Woche lang mit einem Druckverband und verabreichten nicht-steroidale Antirheumatika (NSAR) - Gruppe 1 - oder sie punktierten das Gelenk, zogen die Flüssigkeit ab und versorgten die Patienten dann mit Druckverband und NSAR (Gruppe 2) oder sie schlossen an die Gelenkpunktion noch eine intraartikuläre Kortikoidinjektion an, bevor dieses Kollektiv wie die Vergleichsgruppen Druckverband und NSAR erhielten (Gruppe 3).

Nach vier Wochen war die Bursitis bei allen Patienten verschwunden. Am schnellsten (2,3 Wochen) ging das in Gruppe 3. Am längsten - 3,2 Wochen dauerte es in Gruppe 1. Da die Punktion am Ellenbogen aber komplikationsträchtig ist und der Griff zur Nadel die Erfolgsaussichten offensichtlich nicht wesentlich verbessert, plädieren die Autoren für eine rein konservative Therapie.

- Clin Orthop 2015; online 13. Oktober; doi: 10.1007/s11999-015-4579-0

VIDEO-INTERVIEW AUF SPRINGERMEDIZIN.DE Wie motiviere ich COPD-Patienten zu mehr Bewegung? PD Dr. Henrik Watz, LungenClinic Grosshansdorf, gibt Tipps. $\rightarrow$ www.springermedizin.de/5956368 